\title{
The Value of P504s, 34ßE12, Ki-67 and PSA in Diagnosis and Prognosis of Prostate Cancer
}

Jin-Qi Song, Ya-Nan Zhou,Gang-Liang Tu,Hui Xu

Department of Urology, Affiliated Hospital of Chengde Medical College, Chengde PR China

\begin{abstract}
Background: In recent years, the incidence of prostate cancer(PCa) is increasing. Advanced PCa has a poor prognosis and high cost, which seriously affects the quality of life of patients. Therefore, how to improve the diagnosis rate and prognosis of early PCa is the focus of clinical research. This paper aims to investigate the value of P504s, 34ßE12, Ki-67 and prostate-specific antigen (PSA) in the diagnosis and prognosis of PCa.
\end{abstract}

Objective: To investigate the expression of P504s, 34ßE12, Ki67 and PSA in prostate tissues and their clinical significance.

Methods: Twenty four cases in the study group were selected from PCa confirmed by pathology in the urology department of Chengde Affiliated Hospital from October 2018 to August 2020, and 33 cases of Benign Prostate Hyperplasia (BPH) confirmed by pathology in the same period were selected as the control group.The expression of P504s, 34ßE12 and Ki-67 in prostate tissues were determined by immunohistochemistry.

Results: The positive expression rates of P504s, 34ßE12 and TPSA or F/TPSA in PCa patients were 95.8\%, 12.5\% and $87.5 \%$, respectively. The positive rates in BPH patients were $9.1 \%, 93.9 \%$ and $27.3 \%$, The difference between the two groups was statistically significant $(p<0.05)$. PCa bone or lymph node metastasis was positively correlated with Ki-67 ( $r=0.423, p<0.05)$ and Gleason score $(r=0.446, p<0.05)$, indicating a stronger correlation with Gleason score.

Conclusion: The combined detection of P504s, 34ßE12 and PSA is helpful for the diagnosis and differential diagnosis of PCa.High Gleason score and ki-67 expression may indicate high risk of PCa metastasis and poor prognosis.

Keywords: Prostate Cancer, P504s, 34ßE12, Ki-67, PSA.

\section{Introduction}

In American men, $\mathrm{PCa}$ is the most common nonskin malignant tumor and the second leading cause of death among cancer patients. The incidence rate of PCa in African American men is 62\% higher than that in white men. ${ }^{1} \mathrm{PCa}$ diagnosis rate in China is also increasing year by year, which may be related to aging population, changes in diet structure and living habits as well as improvement of diagnosis and treatment level in medical institutions. Age, race, genetic susceptibility, and demographics are associated with a high incidence of PCa. PCa is highly heterogeneous, with changes ranging from inert tumors to highly invasive tumors. ${ }^{2,3}$ PCa diagnosis, treatment and care place a heavy burden on the global healthcare system. The earlier PCa is found, the better its prognosis will be, because all kinds of therapeutic effects are obvious, and surgical treatment can basically achieve a radical cure effect. The diagnosis and treatment of PCa patients with different degrees of malignancy is a major difficulty, and only active screening can prevent the progression of PCa from the early stage to the incurable stage.At present, there are many test

Correspondence

Hui Xu, Department of Urology, the Affifiliated Hospital of Chengde Medical College, Chengde,Hebei,China, email:chengdexuhui@163.com. 
indexes related to $\mathrm{PCa}$ screening, and single test has its limitations.There are few studies on the relationship between P504s, 34ßE12, Ki-67 and PSA and PCa.In this study, the expression of P504s, 34ßE12, Ki-67 and PSA in prostate tissue was detected to explore the role of P504s, $34 \beta \mathrm{E} 12, \mathrm{Ki}-67$ and PSA in the diagnosis and differential diagnosis of $\mathrm{PCa}$ and to understand its relationship with metastasis risk and prognosis of PCa.

\section{Methods}

Study design and settings: $\mathrm{PCa}$ and $\mathrm{BPH}$ patients admitted to the Urology Department of The Affiliated Hospital of Chengde Medical College from October 2018 to August 2020 were selected.The study plan was approved by the medical Ethics Committee of our hospital, and all patients were aware of and volunteered to participate in this study.

Inclusion criteria: Patients with $\mathrm{PCa}$ and $\mathrm{BPH}$ confirmed by pathological findings and without other malignant tumors.

Exclusion criteria: Acute urinary tract infection patients with other malignant tumors critically ill patients with heart, liver and other viscera diseases patients with complex conditions difficult to make an effective evaluation.

A total of 24 patients in the cancer group, aged $(71.48 \pm 8.10)$ years old, ranged from 58 to 91 years old. And all had Gleason scores. In the hyperplasia group, 33 patients were aged $(70.03 \pm 7.60)$ years, and the age range was 56-89 years. Cancer groups were divided into 3 groups according to Gleason score: Group I (welldifferentiated, Gleason2-6 points), Group II (moderately differentiated, Gleason7 points), and Group III (poorly differentiated/undifferentiated, Gleason $8-10$ points). $3 \mathrm{ml}$ venous blood was extracted from all the subjects and the serum was separated for PSA detection. There was no statistically significant difference in general data between the two groups $(t=0.68, p>0.05)$.

Prostate specimens were taken, fixed in $10 \%$ formaldehyde, paraffin-embedded and sectioned.By immunohistochemical method of P504s, $34 \beta E 12$ and Ki-67were applied.
Interpretation standard: $\mathrm{P} 504 \mathrm{~s}, 34 \beta \mathrm{E} 12$, ki-67 positive results: yellow, brown-yellow, and tan particles appear in the nucleus; if no staining or a small part of staining is seen in the above parts, it will be judged as negative results. TPSA $<4 \mathrm{ng} / \mathrm{ml}$ was judged to be negative, and F/TPSA $<0.16$ when TPSA was $4-10 \mathrm{ng} / \mathrm{ml}$ or TPSA $>$ $10 \mathrm{ng} / \mathrm{ml}$ was judged to be positive .

Statistical analysis: SPSS 22.0 statistical software was used for data analysis. Measurement data were expressed as mean \pm standard deviation, and comparison between groups was performed by $t$ test. The counting data is expressed as percentage and chi-square test is used.Spearman rank correlation analysis was used for correlation analysis. $\mathrm{p}<0.05$ was considered as a statistically significant difference.

\section{Results}

1. Comparison of clinical data between cancer group and hyperplasia group

The positive expression rates of P504s and TPSA or F/ TPSA in the cancer group and the hyperplasia group were $95.8 \%, 87.5 \%, 9.1 \%$ and $27.3 \%$, respectively, with statistically significant differences $(\mathrm{p}<0.05)$. The positive expression rates of $34 \beta \mathrm{E} 12$ were $12.5 \%$ and $93.9 \%$, respectively, with statistically significant differences $(\mathrm{p}<$ 0.05).as shown in table 1.

Table 1:Expression of P504s, 34 E12 and PSA in cancer and hyperplasia groups

\begin{tabular}{|c|c|c|c|c|}
\hline \multirow[t]{2}{*}{ Type } & $\begin{array}{l}\text { Number } \\
\text { of cases }\end{array}$ & P504s & $34 \beta \mathrm{E} 12$ & $\begin{array}{l}\text { TPSA or } \\
\text { F/TPSA }\end{array}$ \\
\hline & $(\mathrm{n}=57)$ & $(+) \quad(-)$ & $(+) \quad(-)$ & $(+) \quad(-)$ \\
\hline $\begin{array}{l}\text { Cancer } \\
\text { group }\end{array}$ & 24 & 23 & 21 & 21 \\
\hline $\begin{array}{l}\text { Hyperplasia } \\
\text { group }\end{array}$ & 33 & 30 & 31 & 24 \\
\hline$x^{2}$ & & 38.72 & 34.98 & 17.87 \\
\hline $\mathrm{p}$ & & $<0.05$ & $<0.05$ & $<0.05$ \\
\hline
\end{tabular}

\section{Relationship between Ki-67 expression and bone or lymph node metastasis}

There were 9 cases in the transfer group, including 3 cases with ki- $67 \leq 10 \%, 5$ cases with $10 \%-30 \%$, and 1 case with $>30 \%$. There were 15 cases in the non-metastatic group, among which 14 cases with ki- $67 \leq 10 \%, 1$ case with $10 \%$ - 
$30 \%$, and 0 cases with $>30 \%$.Correlation analysis of ki- 67 between the transferred group and the non-transferred group showed that the correlation coefficient $r=0.423$ (positive correlation), $\mathrm{p}=0.044<0.05$.PCa bone or lymph node metastasis was correlated with $\mathrm{Ki}-67$ expression to a certain extent, and the higher ki-67 expression was, the higher the risk of bone or lymph node metastasis might be as shown in table 2 .

Table 2: Relationship between Ki-67 expression and bone or lymph node metastasis

\begin{tabular}{|l|c|ccc|}
\hline \multicolumn{1}{|c|}{ Type } & $\begin{array}{c}\text { Number } \\
\text { of cases }\end{array}$ & \multicolumn{3}{|c|}{ Ki-67 } \\
\cline { 2 - 5 } & $(\mathbf{n}=\mathbf{2 4})$ & $\leq \mathbf{1 0} \%$ & $\mathbf{1 0 \% - 3 0 \%}$ & $\leq \mathbf{3 0 \%}$ \\
\hline The transfer group & 9 & 3 & 5 & 1 \\
\hline $\begin{array}{l}\text { The non- } \\
\text { transferred group }\end{array}$ & 15 & 14 & 1 & 0 \\
\hline
\end{tabular}

\section{Gleason score was associated with bone or lymph node metastasis}

There were 9 patients in the metastasis group, including 1 patient in group I, 1 patient in group II and 7 patients in group III.There were 15 patients in the non-metastatic group, including 8 patients in group I, 4 patients in group II and 3 patients in group III. Correlation analysis of Gleason score between the transferred group and the non-transferred group showed that the correlation coefficient $\mathrm{r}=0.446$ (positive correlation), $\mathrm{p}=0.033<0.05$. This indicated that $\mathrm{PCa}$ bone or lymph node metastasis was correlated with Gleason score expression to a certain extent, and the higher the Gleason score was, the higher the risk of bone or lymph node metastasis might be as shown in table 3 .

Table 3: Relationship between Ki-67 expression and bone or lymph node metastasis

\begin{tabular}{|l|l|lcl|}
\hline \multicolumn{1}{|c|}{ Type } & $\begin{array}{c}\text { Number } \\
\text { of cases }\end{array}$ & \multicolumn{3}{|c|}{ Gleasn Score } \\
\cline { 2 - 5 }$(\mathbf{n = 2 4 )}$ & I & II & III \\
\hline The transfer group & 9 & 1 & 1 & 7 \\
\hline $\begin{array}{l}\text { The non-transferred } \\
\text { group }\end{array}$ & 15 & 8 & 4 & 3 \\
\hline
\end{tabular}

\section{Discussion}

Although there are many indicators for tumor detection, single detection has its limitations. In order to improve the sensitivity and specificity of detection, combined detection is the trend and inevitable.P504s, also known as $\alpha$-methylacyl-Coa racemase (AMACR), is a protein in the cytoplasm that can be involved in fatty acid oxidation and derivatives. Its gene is located on chromosome $5 \mathrm{p} .{ }^{13}$ Studies have reported that P504s can be positively expressed in many tumor tissues, and its expression level is closely related to the biological behavior and prognosis of tumors. 4 Studies have shown that P504s is highly sensitive to the diagnosis of $\mathrm{PCa}$, and the higher its expression indicates the higher degree of malignancy of tumor cells, and its high expression is correlated with bone metastasis of PCa. ${ }^{5-6}$ The research results of many scholars showed that the positive expression rate of $\mathrm{P} 504 \mathrm{~s}$ in $\mathrm{BPH}$ patients was lower than that in $\mathrm{PCa}$ patients. ${ }^{7-11} \mathrm{P} 504 \mathrm{~s}$ also has a higher detection rate for $\mathrm{PCa}$ patients with PSA in the early gray zone, and can reduce the number of repeated puncture biopsies. ${ }^{12}$ In this study, there was a significant difference in the expression of P504s in the cancer group and the hyperplasia group, and the results showed that the difference was statistically significant $(\mathrm{P}<0.05)$, consistent with previous research results.34ßE12 was a high molecular weight cytokeratin proposed by Gown in 1984, which was highly expressed in normal prostatic acinar and basal cells. However, most PCa tissues showed basal cell absence, so $34 \beta \mathrm{E} 12$ showed no expression or weak expression in PCa tissues, thus it was regarded as a specific marker of basal cells and play a vital role in the diagnosis and differential diagnosis of benign and malignant prostate diseases. ${ }^{13-15}$ Shah et al reported that PCa was highly suspected as long as the $34 \beta E 12$ staining was negative. ${ }^{16}$ It has also been reported that in atypical adenomatous lesions of the prostate, only some glands are stained positive. However, it has been reported that $34 \beta \mathrm{E} 12$ is positively expressed in squamous cell carcinoma of the renal pelvis (a rare malignancy), so it is expressed differently in different types of malignancies. ${ }^{17}$ In this study, the positive expression rate of $34 \beta \mathrm{E} 12$ was $93.9 \%(31 / 33)$ in the hyperplasia group and $12.5 \%(3 / 24)$ in the cancer group, the difference was statistically significant $(\mathrm{P}<0.05)$. This suggests that $34 \beta \mathrm{E} 12$ may be an effective marker for benign prostatic disease.

PSA is a serine protease that increases sperm motility and liquefies sperm.It is located on chromosome 19q2 and consists of 237 amino acid residues. Physiologically, 
there is a barrier between the prostatic acini and the prostatic lymphatic microcirculation network. When PCa invades capillaries and lymphatic vessel walls, PSA in prostatic acinus will enter into the blood circulation, thus causing serum PSA of PCa patients to rise.The normal on-line level of TPSA is $4 \mathrm{ng} / \mathrm{ml}$, and the gray area is $4-10 \mathrm{ng} / \mathrm{ml}$. Biopsy pathology in the gray area shows that the positive rate of PCa is only $25 \%$, but the positive rate can reach $70 \%$ when TPSA $>10 \mathrm{ng} / \mathrm{ml} .{ }^{18,19}$ In this paper, the positive rate of PCa was $26.7 \%(4 / 15)$ in gray area and $70.8 \%(17 / 24)$ when TPSA $>10 \mathrm{ng} / \mathrm{ml}$. Compared with FPSA and TPSA, F/TPSA has a higher diagnostic value. As for whether PSA patients with gray area need puncture biopsy, the evaluation index of $\mathrm{PCa}$ diagnosis and treatment guide in China is F/TPSA < 0.16.20 PSA and anal examination are often used for early diagnosis and screening of PCa.Although PSA is highly sensitive, its specificity is low. Therefore, patients suspected of $\mathrm{PCa}$ are recommended to undergo multiparameter Magnetic resonance imaging examination and biopsy if necessary. ${ }^{21,22}$ It has been reported that when TPSA > 100ng/ml, it can be confirmed without bone scan and is also believed to have metastasis. The risk threshold of PCa bone metastasis: TPSA > 20ng/ $\mathrm{ml}$, local advanced cancer or Gleason score $\geq 8$, if lower than the threshold, the rate of bone metastasis is considered to be very low. ${ }^{23,24}$ In this study, there were 8 cases with TPSA $>100 \mathrm{ng} / \mathrm{ml}$, and the metastasis rate was $62.5 \%$ (5/8). The positive rates of TPSA or F/TPSA were significantly different between the cancer group and the hyperplasia group $(\mathrm{p}<0.05)$, indicating that PSA can be used in the differential diagnosis of $\mathrm{PCa}$ and BPH.In this paper, a 63-year-old elderly patient was hospitalized twice, for first time: Clinical examination found elevated PSA,TPSA:6.220ng/ml, FPSA:0.441ng/ $\mathrm{ml}(\mathrm{F} / \mathrm{TPSA}<0.16)$ but no obvious abnormality in per rectal examination and on Prostate needle biopsy Benign prostatic hyperplasia was suggested. After discharge, the PSA was rechecked regularly and continued to rise. Then patient was readmitted seven months later with TPSA:9.110ng/ml, FPSA:0.736ng/ml (F/TPSA < 0.16). On anal examination: local uplift of the left lobe, hard texture, disappearance of central sulcus. Prostate needle biopsy came out to be PCa. This may be related to the occurrence of PCa with temporal heterogeneity and tissue heterogeneity. Therefore, for patients with
PSA in gray area and F/TPSA $<0.16$, regular physical examination should be conducted and closely observed. Ki-67 was first discovered by foreign scholars in 1983. It is an antigen that can reflect the proliferative activity of malignant tumor cells and exists in the nucleus with a short half-life. ${ }^{25}$ Its expression level was different in different cell cycles.It was not expressed in the G0 and G1 phases, but gradually increased in the S and G2 phases, reached its peak in the M phase, and disappeared in the later stage of mitosis. ${ }^{26}$ At present, the biological function of Ki-67 protein has not been fully understood. It has been reported that $\mathrm{Ki}-67$ is not expressed in benign prostate tissues, but is highly expressed in $\mathrm{PCa}$ tissues.It can predict the prognosis of $\mathrm{PCa}{ }^{27-29}$ It has been reported by foreign scholars that $93 \mathrm{PCa}$ patients received preoperative diffusion-weighted imaging and postoperative immunohistochemistry was used to evaluate Ki-67. The results confirmed that apparent diffusion coefficient can be used as a non-invasive indicator to evaluate $\mathrm{Ki}-67$ and PCa invasiveness, which is of great significance for PCa pathological grading and prognosis assessment. ${ }^{30,31}$ In this study, there was a certain correlation between PCa bone or lymph node metastasis and Ki-67 expression and Gleason score, indicating that Ki-67 and Gleason score could be used as indicators to evaluate the metastasis risk and prognosis of $\mathrm{PCa}$.

\section{Conclusion}

Patients with advanced PCa have poor prognosis and high cost. So far, the etiology of PCa is still unclear, so the key to improving prognosis lies in early detection, diagnosis and treatment. In particular, BPH patients with PSA in gray area and F/TPSA $<0.16$ should be actively reviewed. Combined detection of P504s, 34ßE12 and PSA can make up for the shortcomings of single detection, which is beneficial to the early diagnosis and differential diagnosis of PCa. Gleason score and Ki-67 can evaluate the metastasis risk and prognosis of PCa. Due to the limited number of cases in this study and the temporal and histological heterogeneity of $\mathrm{PCa}$, more experiments are needed to verify the accuracy of the experimental results. There is still a long way to go in the study of malignant tumors. It is believed that with the progress of technology and in-depth study on the mechanism of action of various detection indicators, more harvest will be gained in the aspects of tumor 
occurrence and development, invasion and metastasis, pathological grading, clinical treatment and prognosis assessment etc.

\section{References}

1. Schymura MJ, Sun L, Percy-Laurry A. Prostate cancer collaborative stage data items-their definitions, quality, usage, and clinical implications:a review of SEER data for 2004-2010. Cancer. 2014, 20 (23):3758-3770.

2. Stelcer E, Konkol M, Suchorska WM, et al. Liquid biopsy in oligometastatic prostate cancer-a biologist's point of view. Front Oncol. 2019, 421(9):775-786.

3. Jemal A, Fedewa SA, Ma J, et al. Prostate cancer incidence and PSA testing patterns in relation to USPSTF screening recommendations. Jama. 2015, 314(19):2054-2061.

4. Senol S, Ceyran A, Kfsemetin D, et a1. Immunohistochemical profile of tumor pathways and prognostic significance in colon adenocarcinomas. J Environ Pathol Toxicol Oncol. 2017, 36(1):29-41.

5. Zhang PX. Correlation between P504S expression and Gleason score in prostate cancer and bone metastasis. Xinjiang medical university. 2013, 15(6):1-55.

6. Cai B, Wang L, Li HM. Expression of P504S/AMACR in prostate cancer specimen puncture and its clinical significance. Modern Oncology. 2012, 26(3):423426.

7. Wu NL. Expression of P504s and P63 in PSA gray area prostate cancer and their clinical value in relation to F/TPSA and PSAD. Healthful Friend. 2020, 13(7):269.

8. Zhang JJ, Shi XX, Tao LL, et al. Expression of FOXA1 and AMACR/P504s in prostate cancer and its clinical significance. China Modern Medicine. 2019, 26(26):7-10.

9. Li L, Zhu WY, Tao ZY, et al. Expression of P504s in prostate cancer. China Modern Doctor. 2017, 55(29):6-8,26,169.

10. Mo Z, Liao JL, Wan L. Expression and clinical significance of PSCA and P504S in patients with prostate cancer. Clinical Medical \& Engineering. 2015, 25(9):1187-1188.

11. Wang K, Qu HC, Xie QP, et al. Changes of P504S protein expression in prostate cancer and its clinical significance. Anti-Tumor Pharmacy. 2019, 9(1):9093.

12. Li L. Expression of PSMA and P504s in PCa. Bengbu Medical College. 2018.

13. Gown AM, Vogel AM. Monoclonal antibodies to human intermediate filament Proteins. Am J Pathol. 1984, 114(2):309-312.

14. Li GC. The significance of P504s, P63, PSMA and Ck34ßE12 in the differentiation of benign and malignant prostate lesions. Surgical Research and New Technique. 2012, 7(2):93-96.

15. Li YJ, Zhang CL, Yang YL, et al. Expression and clinical significance of TMPRSS2-ERG, P504S, P63 and $34 \beta \mathrm{E} 12$ in prostate cancer. Journal of Yanan University (Med Sci ). 2017, 15(2):22-24.

16. Shah IA, Schlageteer MO, Stinnett P, et al. Cytokeratin immunohistochemuisry as a diagnostic tool for distinguishing malignant from benign epithelial lesions of the prostate. Mod Pathol. 1991, 4 (1) : 220224.

17. Huang JW, Cai XY, Wang ZY, et al. Analysis of diagnosis and treatment of renal pelvis squamous cell carcinoma. Chin J Urology. 2020, 41(5):348-351.

18. Makarov DV, Loeb S, Getzenberg RH, et al. Biomarkers for prostate cancer. Annu Rev Med. 2009, 60:139-151.

19. Li HB, Zou XX, Liao QS, et al. The expression and significance of serum PSA, F-PSA and HE4 in prostate cancer. The Practical Journal of Cancer. 2020, (5):724-727.

20. Huang ST, Pan CZ, Tang SL, et al. The diagnostic value of free Prostate specific antigen ratio combined with Prostate specific antigen density in PSA $<4.0 \mathrm{ng} /$ $\mathrm{ml}$ prostate cancer. Journal of Qiqihar University of medicine. 2020, 41(2):161-163.

21. ElifG,Emre E, Mahmut K. Evaluation of relationships between the final Gleason score, PI-RADS v2 score, ADC value, PSA level, and tumor diameter in patients that underwent radical prostatectomy due to prostate cancer. La radiologia medica. 2020, 125(9):827-837.

22. Zlata $\mathrm{H}$, Jana $\mathrm{K}$, Zdenek $\mathrm{K}$. The percentage of free PSA and urinary markers distinguish prostate cancer from benign hyperplasia and contribute to a more 
accurate indication for prostate biopsy. J Biomed Biotechnol. 2020, 8 (6) : 173-185.

23. Pron G. Prostate-specific antigen(PSA)-based population screening for prostate cancer: An evidence-based analysis. Ontario Health Technology Assessment Series. 2015, 15(10):1-12.

24. Wang YL, Hong H, Wu GZ, et al. Correlation analysis of prostate calcification, PSA, Gleason score and bone metastasis of prostate cancer. Chinese J Ultrasound Med. 2020, 36(5):458-462.

25. Gerdes J, Schwab U, Lemke H, et al. Production of a mouse monoclonal antibody reactive with a human nuclear antigen associated with cell proliferation. $\mathrm{Nt}$ J Cancer. 1983, 31 (1) : 13-20.

26. Xu XL. The relationship between the expression of P504s, P63 and Ki-67 in prostate cancer tissues and the prognosis and metastasis of prostate cancer. Wannan Medical College. 2016.

27. Guo JN. Expression and correlation study of Ki-67, P504S and P63 in prostate cancer. Jinan University.
2011.

28. Zhang JW, Zhao YY, Ma AL, et al. Expression of Ki67, P504s, 34ßE12 and P63 in prostate cancer. Ningxia Med J. 2008, 40(10):921-923.

29. Mariarosa P, Cinzia A, Renzo B, et al. The proliferation marker Ki67, but not neuroendocrine expression, Is an independent factor in the prediction of prognosis of primary cancer patients. Radiol Oncol. 2016, 50(3):313-320.

30. Akbari M, Avakoli MB, Izadpanahi $M$, et al. Evaluation of correlation of Ki-67 factor in prostate cancer with apparent diffusion coefficient index in multi b-value diffusion magnetic resonance Imaging (Article). J Nippon Med Sch. 2019, 37(547):11621168.

31. Zhang J, He Y. Ki-67 proliferative index in patients with prostate cancer following radical prostatectomy and its prognostic value(Article). Journal of Practical Oncology. 2019, 34(1):16-22. 